\title{
Structural, thermodynamic, and local probe investigations of the honeycomb material $\operatorname{Ag}_{3} \operatorname{LiMn}_{2} \mathrm{O}_{6}$
}

\author{
R. Kumar, ${ }^{1}$ Tusharkanti Dey, ${ }^{2}$ P. M. Ette, ${ }^{3}$ K. Ramesha, ${ }^{3}$ Atasi Chakraborty, ${ }^{4}$ I. Dasgupta, ${ }^{4}$ R. Eremina, ${ }^{5,6}$ Sándor Tóth, \\ A. Shahee, ${ }^{1}$ S. Kundu, ${ }^{1}$ M. Prinz-Zwick, ${ }^{8}$ A. A. Gippius, ${ }^{9,}{ }^{10}$ H. A. Krug von Nidda, ${ }^{8}$ N. Büttgen, ${ }^{8}$ P. Gegenwart, ${ }^{2}$ \\ and A. V. Mahajan ${ }^{1, *}$ \\ ${ }^{1}$ Department of Physics, Indian Institute of Technology Bombay, Powai, Mumbai 400076, India \\ ${ }^{2}$ Experimental Physics VI, Center for Electronic Correlations and Magnetism, University of Augsburg, D-86159 Augsburg, Germany \\ ${ }^{3}$ Central Electrochemical Research Institute-Madras Unit, CSIR-Madras Complex, Taramani, Chennai 600113, India \\ ${ }^{4}$ School of Physical Sciences, Indian Association for the Cultivation of Science, Jadavpur, Kolkata 700 032, India \\ ${ }^{5}$ Kazan (Volga Region) Federal University, Kremlevskaya Street, 18, Kazan 420008, Russia \\ ${ }^{6}$ Kazan E. K. Zavoisky Physical-Technical Institute (KPhTI) of the Kazan Scientific Center of the Russian Academy of Sciences, Sibirsky tract, \\ 10/7, Kazan 420029, Russia \\ ${ }^{7}$ Laboratory for Neutron Scattering and Imaging, Paul Scherrer Institute (PSI), CH-5232 Villigen, Switzerland \\ ${ }^{8}$ Experimental Physics V, Center for Electronic Correlations and Magnetism, University of Augsburg, D-86159 Augsburg, Germany \\ ${ }^{9}$ Department of Physics, M. V. Lomonosov Moscow State University, 199991 Moscow, Russia \\ ${ }^{10}$ P. N. Lebedev Physics Institute of Russian Academy of Science, 199991 Moscow, Russia
}

(Received 18 January 2019; published 30 April 2019)

\begin{abstract}
Here we present the structural and magnetic properties of the new honeycomb material $\operatorname{Ag}_{3} \operatorname{LiMn}_{2} \mathrm{O}_{6}$. The system $\mathrm{Ag}\left[\mathrm{Li}_{1 / 3} \mathrm{Mn}_{2 / 3}\right] \mathrm{O}_{2}$ belongs to a quaternary $3 R$-delafossite family and crystallizes in a monoclinic symmetry with space group $C 2 / m$, and the magnetic $\mathrm{Mn}^{4+}(S=3 / 2)$ ions form a honeycomb network in the $a b$ plane. An anomaly around $50 \mathrm{~K}$ and the presence of antiferromagnetic (AFM) coupling (Curie-Weiss temperature $\theta_{C W} \sim-51 \mathrm{~K}$ ) were inferred from our magnetic susceptibility data. The magnetic specific heat clearly manifests the onset of magnetic ordering in the vicinity of $48 \mathrm{~K}$, and the recovered magnetic entropy, above the ordering temperature, falls short of the expected value, implying the presence of short-range magnetic correlations. An asymmetric Bragg peak (characteristic of two-dimensional order), seen in neutron diffraction, gains intensity even above the ordering temperature, thus showing the existence of short-range spin correlations. Our electron spin resonance (ESR) experiments corroborate the bulk magnetic data. Additionally, the ESR line broadening upon approaching the ordering temperature $T_{\mathrm{N}}$ could be described in terms of a BerezinskiiKosterlitz-Thouless scenario with $T_{\mathrm{KT}}=40(1) \mathrm{K}$. The ${ }^{7} \mathrm{Li}$ NMR line shift probed as a function of temperature tracks the static susceptibility $\left(K_{\text {iso }}\right)$ of magnetically coupled $\mathrm{Mn}^{4+}$ ions. The ${ }^{7} \mathrm{Li}$ spin-lattice relaxation rate $\left(1 / T_{1}\right)$ exhibits a sharp decrease below about $50 \mathrm{~K}$. A critical divergence is absent at the ordering temperature perhaps because of the filtering out of the antiferromagnetic fluctuations at the Li site, i.e., at the centers of the hexagons in the honeycomb network. Combining our bulk and local probe measurements, we establish the presence of an ordered ground state for the honeycomb system $\mathrm{Ag}_{3} \mathrm{LiMn}_{2} \mathrm{O}_{6}$. Our ab initio electronic structure calculations suggest that in the $a b$ plane, the nearest-neighbor $(\mathrm{NN})$ exchange interaction is strong and AFM, while the next-NN and the third-NN exchange interactions are FM and AFM, respectively. The interplanar exchange interaction is found to be relatively small. In the absence of any frustration the system is expected to exhibit long-range, AFM order, in agreement with experiment.
\end{abstract}

DOI: 10.1103/PhysRevB.99.144429

\section{INTRODUCTION}

Materials based on the delafossite structure with the general chemical formula $A B \mathrm{O}_{2}$ exhibit interesting physical properties [1-5]. In general, $A$ and $B$ sites in $A B \mathrm{O}_{2}$ represent monovalent and trivalent cations, respectively, having a linear and octahedral environment of oxygen atoms. In this case, the $B$ site that is responsible for magnetism in delafossite materials forms an edge-shared triangular lattice or a honeycomb lattice when the system crystallizes in hexagonal/rhombohedral or monoclinic space groups, respectively.

\footnotetext{
*Corresponding author: mahajan@phy.iitb.ac.in
}

There exists a variety of honeycomb materials [6] and in recent years attempts have been made to synthesize delafossite materials with tetravalent ions at the $B$ site (honeycomb lattice), which are known as $3 R$-delafossites. A few examples of $3 R$-delafossites are $\mathrm{Ag}\left(\mathrm{Li}_{1 / 3} \mathrm{Ru}_{2 / 3}\right) \mathrm{O}_{2}$ [7-9], $\operatorname{Ag}\left(\mathrm{Li}_{1 / 3} \mathrm{Rh}_{2 / 3}\right) \mathrm{O}_{2}$ [10], and $\mathrm{Ag}\left(\mathrm{Li}_{1 / 3} \mathrm{Ir}_{2 / 3}\right) \mathrm{O}_{2}$ [10]. We would like to mention here that $\mathrm{Ag}$ insertion into the primary structure of $\mathrm{Li}_{2} M \mathrm{O}_{3}(M=\mathrm{Ru}, \mathrm{Rh}$, or Ir) results in its placement between consecutive metal layers, which essentially reduces the interlayer connectivity and thus makes the materials highly two-dimensional (2D) in nature.

As per some recent theoretical studies [11-13] novel ground state properties are expected for $4 d / 5 d$ materials depending on the variation of superexchange energy scale, 
Hund's coupling $\left(J_{H}\right)$, and spin-orbit coupling (SOC). In particular, honeycomb-lattice-based $5 d$ materials are at the forefront of current experimental and theoretical research because of the possibility of stabilizing the Heisenberg-Kitaev Hamiltonian, and a rich phase diagram upon variation of the exchange couplings is envisaged [14]. On the other hand, a different scenario might emerge while dealing with $3 d$ transition metal ions, where SOC is much weaker than the other energy scales, namely $U$ (on-site Coulomb repulsion) and $J_{H}$. For instance, it has recently been shown by Wei et al. [15] that the 2D-honeycomb-lattice-based Affleck-KennedyLieb-Tasaki (AKLT) state with $S=3 / 2$ is a prototype for the physical realization of the measurement-based quantum computation (MBQC). According to Maciej et al. [16] the multiorbital insulators in the framework of Hubbard models with nearest-neighbor hopping on a honeycomb lattice could even lead to the $S=3 / 2$ AKLT state. In light of the aforementioned proposals, it is worth investigating the physical properties of a honeycomb lattice with $S=3 / 2$, with the intention of exploring some novel ground state properties.

Herein, we report the sample preparation and structural and physical properties of the $3 d$ transition metal oxide (delafossite) $\mathrm{Ag}\left(\mathrm{Li}_{1 / 3} \mathrm{Mn}_{2 / 3}\right) \mathrm{O}_{2}$. In this compound, the $\mathrm{Mn}^{4+}$ ions $(S=3 / 2)$ form a $2 \mathrm{D}$ honeycomb lattice with $\mathrm{Li}$ ions positioned at the center of each honeycomb unit. The material was structurally characterized by a combination of x-ray and neutron diffraction measurements, along with magnetization, specific heat, and electron spin resonance (ESR). In addition, ${ }^{7} \mathrm{Li}$ nuclear magnetic resonance (NMR) spectra and spinlattice relaxation rate measurements were performed. Structural characterization done with $\mathrm{x}$-ray and neutron diffraction suggest a superstructure (honeycomb) formed by magnetic $\mathrm{Mn}^{4+}$ ions in the crystallographic $a b$ plane. Interestingly, the same asymmetric peak, seen in the paramagnetic region, is found to become more intense upon lowering the temperature, as observed in our neutron diffraction studies. Magnetization data show an anomaly in the vicinity of $50 \mathrm{~K}$ and antiferromagnetic coupling is inferred from our susceptibility analysis. Another thermodynamic measurement, specific heat, also confirms this anomaly and locates the magnetic ordering at $47 \mathrm{~K}$. The integrated intensities of the electron spin resonance (ESR) absorption lines mimic the bulk magnetic susceptibility. The ESR measurements evidence a critical broadening as a function of temperature with a transition temperature $T_{\mathrm{N}}=45 \mathrm{~K}$. Further, the line broadening upon approaching $T_{\mathrm{N}}$ may be alternatively described in terms of a BerezinskiiKosterlitz-Thouless (BKT) scenario with $T_{\mathrm{KT}}=40(1) \mathrm{K}$. The static susceptibility (free from defects/impurities) tracked using local probe ${ }^{7} \mathrm{Li}$ NMR spectra measurements also reproduces the anomaly observed in bulk susceptibility measurements, while the ${ }^{7} \mathrm{Li}$ spin-lattice relaxation rate does not show any sharp anomaly around the $50 \mathrm{~K}$ transition. This is likely due to the symmetric location of the ${ }^{7} \mathrm{Li}$ with respect to the magnetic $\mathrm{Mn}^{4+}$ ions, giving rise to a filtering of antiferromagnetic fluctuations. Our experimental results corroborate the establishment of a magnetically ordered ground state for the $3 d$-based system $\operatorname{Ag}\left(\mathrm{Li}_{1 / 3} \mathrm{Mn}_{2 / 3}\right) \mathrm{O}_{2}$, which is also supported by first-principles electronic structure calculation.

\section{EXPERIMENTAL AND COMPUTATIONAL DETAILS}

The polycrystalline samples of the quaternary $3 R$ delafossite oxide $\mathrm{Ag}\left[\mathrm{Li}_{1 / 3} \mathrm{Mn}_{2 / 3}\right] \mathrm{O}_{2}$ were prepared by a combination of sol-gel and ion-exchange methods. First, the starting material $\mathrm{Li}_{2} \mathrm{MnO}_{3}$ was prepared by sol-gel route and then fired at $500^{\circ} \mathrm{C}$ for 6 hours. After confirming by x-ray diffraction that $\mathrm{Li}_{2} \mathrm{MnO}_{3}$ was single phase, $\mathrm{AgNO}_{3}$ was mixed with $\mathrm{Li}_{2} \mathrm{MnO}_{3}$ in the ratio $1: 3$. The resultant mixture was slowly heated to $300{ }^{\circ} \mathrm{C}$ and held for 6 hours following which the desired material $\mathrm{Ag}\left[\mathrm{Li}_{1 / 3} \mathrm{Mn}_{2 / 3}\right] \mathrm{O}_{2}$ was obtained by removing the residual by-product $\mathrm{LiNO}_{3}$ by washing the mixture with water.

The room temperature powder $\mathrm{x}$-ray diffraction $(\mathrm{XRD})$ measurements were performed using a PANalytical Xpert Pro x-ray diffractometer with $\mathrm{Cu}-\mathrm{K}_{\alpha}$ radiation $(\lambda=1.5418 \AA)$. Neutron diffraction data were taken on the DMC beamline at the Paul Scherrer Institute (PSI) using a wavelength $\lambda=$ $2.4586 \AA$. Microstructural investigations were performed with a CM 200 Philips transmission electron microscope (TEM) operating at $200 \mathrm{kV}$. Magnetization $M$ measurements in the temperature range $2-400 \mathrm{~K}$ as a function of applied field $H$ were performed on a Quantum Design SQUID VSM with the powder sample loaded in a capsule, and for measurements in the 400-630 K range, the high-temperature option of the Quantum Design VSM was used. The heat capacity measurements were carried out on a Quantum Design PPMS using the thermal-relaxation method. ESR was measured in an ELEXSYS E500 spectrometer (Bruker) at X-band frequency of $9.4 \mathrm{GHz}$ in a magnetic field of about $H=18 \mathrm{kOe}$. The spectrometer was equipped with a helium gas-flow cryostat ESR 900 (Oxford Instruments) operating in the temperature range $T=4-300 \mathrm{~K}$. The polycrystalline samples were immersed in paraffin in suprasil quartz glass tubes and mounted in the cavity. ESR detects the microwave absorption due to magnetic dipolar transitions induced between the Zeeman levels of the sample as a function of the external magnetic field. Due to the lock-in amplification technique by field modulation in ESR, one records the field derivative of the absorption spectra. The ${ }^{7} \mathrm{Li}$ NMR measurements (spectra and spin-lattice relaxation rate $1 / T_{1}$ ) were performed both in the fixed-field $(93.9543 \mathrm{kOe})$ and swept-field $(v=95 \mathrm{MHz})$ modes to gain further insights into the intrinsic static susceptibility of $\mathrm{Mn}$ moments in $\mathrm{Ag}_{3} \mathrm{LiMn}_{2} \mathrm{O}_{6}$ by measuring the line shift as a function of temperature.

All the electronic structure calculations based on DFT presented in this paper were carried out in the plane-wave basis within the generalized gradient approximation (GGA) [17] of the Perdew-Burke-Ernzerhof exchange correlation supplemented with Hubbard $U$ as encoded in the Vienna $a b$ initio simulation package (VASP) $[18,19]$ with projector augmented wave potentials $[20,21]$. The calculations are done with usual values of $U$ and Hund's coupling $\left(J_{H}\right)$ chosen for Mn with $U_{\text {eff }}\left(\equiv U-J_{H}\right)=3.0 \mathrm{eV}$ in the Dudarev scheme [22]. In order to achieve convergence of energy eigenvalues, the kinetic energy cutoff of the plane wave basis was chosen to be $600 \mathrm{eV}$. The Brillouin zone integrations are performed with an $8 \times 4 \times 6$ Monkhorst grid of $k$ points. The exchange paths were identified by calculating hopping parameters by constructing the Wannier function using the 


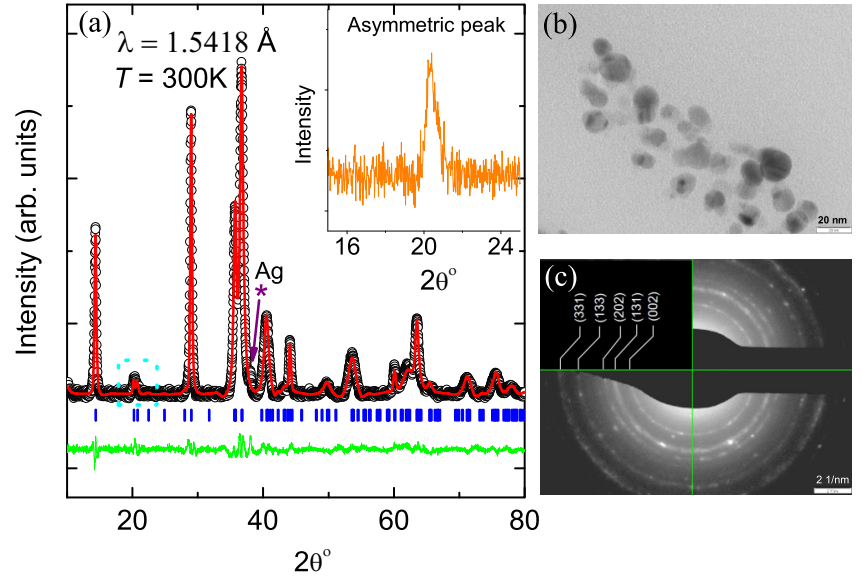

FIG. 1. (a) X-ray diffraction pattern for $\mathrm{Ag}_{3} \mathrm{LiMn}_{2} \mathrm{O}_{6}$ with $\lambda=$ $1.5418 \AA$ at $300 \mathrm{~K}$. Experimental data (black open circles) and Rietveld refined data (red solid line) are shown. The Bragg positions and the difference pattern are shown by blue vertical lines and green line, respectively. The asymmetric peak seen around $21^{\circ}$ is marked with a dotted rectangular box and inset shows the magnified version of the portion in the box. The peak marked with an asterisk (*) is an impurity peak due to unwashed $\mathrm{Ag}$ remaining in the final product. (b) TEM image of $\mathrm{Ag}_{3} \mathrm{LiMn}_{2} \mathrm{O}_{6}$ nanoparticles. (c) Selected area electron diffraction (SAED) of $\mathrm{Ag}_{3} \mathrm{LiMn}_{2} \mathrm{O}_{6}$ nanoparticles, where the $(h k l)$ indexing of various rings is shown.

VASP2WANNIER and WANNIER90 codes [23]. In addition, to get the minimum-energy structure, symmetry-protected ionic relaxation was carried out using the conjugate-gradient algorithm until the Hellman-Feynman forces on each atom were less than the tolerance value of $0.01 \mathrm{eV} / \AA$.

\section{RESULTS AND DISCUSSION}

We will now present the results of our various bulk and local probe measurements on $\mathrm{Ag}_{3} \mathrm{LiMn}_{2} \mathrm{O}_{6}$.

\section{A. Structure analysis}

Figure 1 depicts the $\mathrm{x}$-ray diffraction pattern for $\mathrm{Ag}_{3} \mathrm{LiMn}_{2} \mathrm{O}_{6}$ at $300 \mathrm{~K}$. The $\mathrm{x}$-ray diffraction pattern for $\mathrm{Ag}_{3} \mathrm{LiMn}_{2} \mathrm{O}_{6}$ was found to be similar to the isostructural material $\mathrm{Ag}_{3} \mathrm{LiRu}_{2} \mathrm{O}_{6}$ [7] of the quaternary $3 R$-delafossite family. A peak corresponding to a small amount of $\mathrm{Ag}$ is seen in the XRD pattern. An asymmetric reflection (a superstructure peak) was also seen around $2 \theta \approx 21^{\circ}$ (see the inset of Fig. 1). This asymmetric peak (more prominent in neutron diffraction), commonly known as the Warren peak [24], arises as a consequence of the irregular stacking sequences of layers in a structure. In the present case, $\mathrm{Mn}^{4+}$ ions are found to form $2 \mathrm{D}$ honeycomb layers in the $a b$ plane and the irregular stacking pattern results from the stacking faults, which then limit the correlation length in the crystallographic $c$ direction. The x-ray diffraction peaks for $\mathrm{Ag}_{3} \mathrm{LiMn}_{2} \mathrm{O}_{6}$ were found to be broader than those of $\mathrm{Ag}_{3} \mathrm{LiRu}_{2} \mathrm{O}_{6}$, and the particle size determined from the Scherrer formula is estimated to be about $20 \mathrm{~nm}$. We then performed microstructure analysis from the TEM and selected area electron diffraction (SAED) images depicted in Fig. 1. SAED analysis shows that the continuous
TABLE I. The structural parameters obtained after Rietveld refinement of the x-ray diffraction data collected at a wavelength of $\lambda=1.5418 \AA$ for $\mathrm{Ag}_{3} \mathrm{LiMn}_{2} \mathrm{O}_{6}$ under the space group $C 2 / m$ at $300 \mathrm{~K}$. The obtained lattice constants are $a=5.0690(7) \AA, b=$ 8.7863(11) $\AA$, and $c=6.3755(9) \AA$ with $\beta=74.938(11)^{\circ}$.

\begin{tabular}{lcccccc}
\hline \hline Atoms & Site & $x$ & $y$ & $z$ & $B_{\text {iso }}$ & Occ. \\
\hline $\mathrm{Ag} 1$ & $2 \mathrm{~d}$ & 0 & 0.5 & 0.5 & 0.52 & 1 \\
$\mathrm{Ag} 2$ & $4 \mathrm{~h}$ & 0.5 & $0.3271(4)$ & 0.5 & 0.52 & 1 \\
$\mathrm{Li}$ & $2 \mathrm{a}$ & 0 & 0 & 0 & 0.49 & 1 \\
$\mathrm{Mn}$ & $4 \mathrm{~g}$ & 0 & $0.6648(11)$ & 0 & 0.45 & 1 \\
$\mathrm{O} 1$ & $8 \mathrm{j}$ & $0.4270(23)$ & $0.3471(17)$ & $0.8420(18)$ & 0.36 & 1 \\
$\mathrm{O} 2$ & $4 \mathrm{i}$ & $0.1459(45)$ & 0.5 & $0.1393(36)$ & 0.36 & 1 \\
\hline \hline
\end{tabular}

ring patterns [see inset of Fig. 1(c)] from our polycrystalline sample could be well indexed with the hexagonal lattice of $\mathrm{Ag}_{3} \mathrm{LiMn}_{2} \mathrm{O}_{6}$. Our TEM analysis showed that the mean size of nanoparticles was between 10-20 $\mathrm{nm}$ and the nanoparticles appeared to be nearly spherical in morphology. This value is in good agreement with results obtained from XRD. The lowtemperature synthesis route of the starting material $\mathrm{Li}_{2} \mathrm{MnO}_{3}$ (see experimental section) could possibly be the reason for the nanocrystallinity of this material.

In order to extract information about the unit cell parameters and atomic positions, the $\mathrm{x}$-ray diffraction pattern of $\mathrm{Ag}_{3} \mathrm{LiMn}_{2} \mathrm{O}_{6}$ was refined under the FullProf Suite program [25] using the structural parameters of $\mathrm{Ag}_{3} \mathrm{LiRu}_{2} \mathrm{O}_{6}$ as an initial model. All the Bragg reflections obtained for $\mathrm{Ag}_{3} \mathrm{LiMn}_{2} \mathrm{O}_{6}$ could be successfully indexed with a monoclinic space group $C 2 / m$ and the refined atomic coordinates and lattice constants are listed in Table I. Because of the particle size being in the nanometer range, evident from the broadened $\mathrm{x}$-ray peaks and TEM images, microstructure parameters were also taken into account while refining the crystal structure, and a quadratic form of strain formation under Laue class $\mathrm{mmm}$ was considered. The Rietveld refinement quality factors expressed by $R_{w p}, R_{\text {exp }}, R_{p}$, and $\chi^{2}$ have the values $2.89 \%, 1.82 \%, 2.22 \%$, and 2.52 , respectively. The crystal structure of $\mathrm{Ag}_{3} \mathrm{LiMn}_{2} \mathrm{O}_{6}$ based on x-ray diffraction refinement is shown in Fig. 2. The $\mathrm{MnO}_{6}$ octahedra form an edge-sharing, 2D, honeycomb network in the crystallographic $a b$ plane, and the $\mathrm{LiO}_{6}$ octahedra sit at the center of the honeycomb network; see Figs. 2(b) and 2(c). Intercalated Ag atoms go in between the consecutive honeycomb layers.

In our neutron diffraction data (see the inset of Fig. 3), an asymmetric peak is seen to emerge below about $50 \mathrm{~K}$ while all the other peaks are nearly unchanged. This must be from the ordering transition that is evident in other measurements such as magnetic susceptibility and heat capacity, which are detailed in the following sections.

\section{B. Magnetization}

Figure 4 depicts the dc susceptibility $(M / H)$ of $\mathrm{Ag}_{3} \mathrm{LiMn}_{2} \mathrm{O}_{6}$ measured in the temperature range 2-630 K with an applied field of $30 \mathrm{kOe}$. The susceptibility shows a gradual increase upon lowering the temperature before exhibiting a well-rounded anomaly at around $50 \mathrm{~K}$, following which it exhibits an upturn. The anomaly observed at 


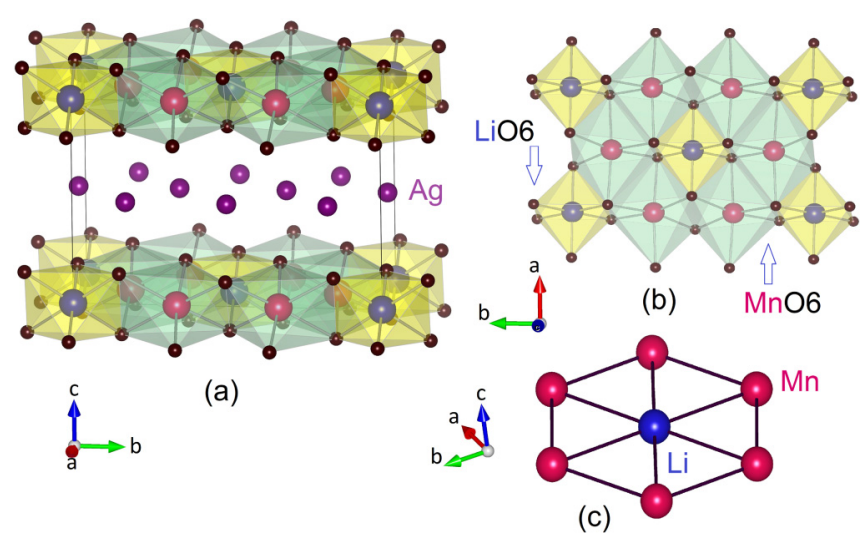

FIG. 2. (a) Unit cell of $\mathrm{Ag}_{3} \mathrm{LiMn}_{2} \mathrm{O}_{6}$ in a monoclinic symmetry with space group $C 2 / m$. (b) Formation of edge-sharing $\mathrm{LiO}_{6} / \mathrm{MnO}_{6}$ octahedra in $a b$ plane. (c) $2 \mathrm{D}$ honeycomb lattice of $\mathrm{Mn}$ atoms having $\mathrm{Li}$ atom at its center.

$50 \mathrm{~K}$ might be a signature of magnetic order, while the low- $T$ increase of susceptibility could be partly due to some extrinsic contributions and/or defects (see ESR/NMR results later in the paper). The Curie-Weiss fit $\left[\chi=\chi_{0}+C /\left(T-\theta_{C W}\right)\right]$ to the susceptibility data in the temperature range $240-630 \mathrm{~K}$ yields temperature-independent susceptibility $\chi_{0}=$ $-7.835 \times 10^{-4} \mathrm{~cm}^{3} / \mathrm{mol} \mathrm{Mn}$, the Curie-Weiss temperature $\theta_{C W} \sim-51(1) \mathrm{K}, \quad$ indicative of antiferromagnetic interactions among $\mathrm{Mn}^{4+}$ moments, and a Curie constant $C=2.495 \pm 0.014 \mathrm{~cm}^{3} \mathrm{~K} / \mathrm{mol} \quad \mathrm{Mn} \quad$ or an effective paramagnetic moment $\sim 4.46 \pm 0.33 \mu_{B}$. The electronic configuration of $\mathrm{Mn}^{4+}$ is $3 d^{3}$ and hence the expected effective moment (considering $g=2$ as obtained from electron spin resonance discussed later) is $3.87 \mu_{B}$. Note that the value of

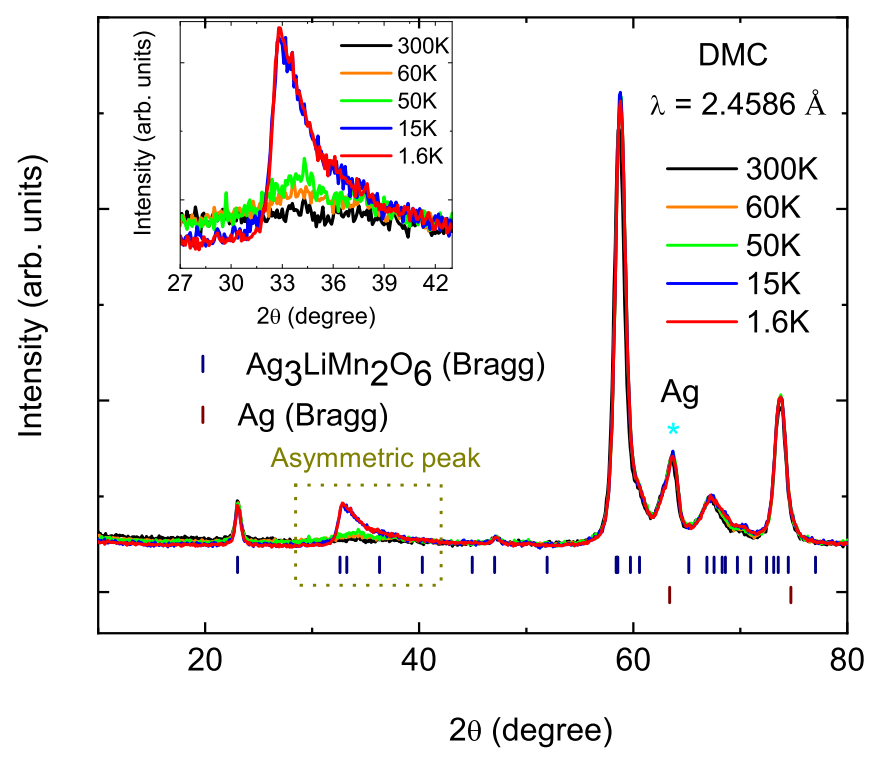

FIG. 3. Neutron diffraction data collected for $\mathrm{Ag}_{3} \mathrm{LiMn}_{2} \mathrm{O}_{6}$ with $\lambda=2.4586 \AA$ at different temperatures. Vertical green and black lines represent the Bragg positions for $\mathrm{Ag}_{3} \mathrm{LiMn}_{2} \mathrm{O}_{6}$ and the impurity phase $\mathrm{Ag}$, respectively. An enhancement in the intensity of peak at $33^{\circ}$ is due to the appearance of magnetic order. The impurity peak of $\mathrm{Ag}$ is marked with an asterisk.

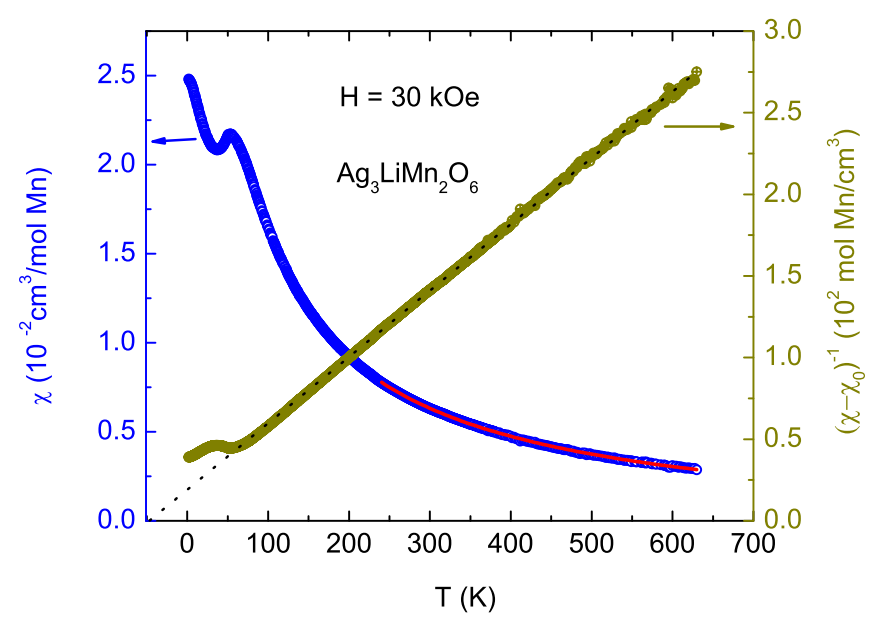

FIG. 4. Left $y$ axis: Variation of the susceptibility in the temperature range $2-630 \mathrm{~K}$ with an applied field of $30 \mathrm{kOe}$ and the Curie-Weiss fit (red line) in the temperature range 240-630 K. Right $y$ axis: Inverse susceptibility, free from temperature-independent susceptibility $\left(\chi_{0}\right)$, in the temperature range $2-630 \mathrm{~K}$. The intercept (dotted black line) on the $x$ axis gives a Curie-Weiss temperature of about $-51 \mathrm{~K}$.

$\chi_{0}$ cannot be determined very accurately as the susceptibility has still not leveled off even above $600 \mathrm{~K}$. Consequently, there is some uncertainty in the determination of the Curie constant and $\theta_{C W}$. Further, 10-20 nm size grains are present in our sample. Defects on the surfaces of nanoparticles could also stabilize a moment and contribute to the observed value. A higher than expected effective moment was also observed in $\mathrm{CaMnO}_{3}$, which increased with oxygen depletion [26].

\section{Specific heat}

The specific heat of the cold pressed powder sample of $\mathrm{Ag}_{3} \mathrm{LiMn}_{2} \mathrm{O}_{6}$ was measured and an anomaly was also noticed there as was observed in our magnetization data. Figure 5(a) depicts the temperature variation of specific heat $C_{p}(T)$ of $\mathrm{Ag}_{3} \mathrm{LiMn}_{2} \mathrm{O}_{6}$ in the temperature range $1.8-200 \mathrm{~K}$ in 0 and 90 kOe magnetic fields. The $C_{p}(T)$ data distinctly show the presence of an anomaly around $48 \mathrm{~K}$, which is found to be insensitive to the applied magnetic field. The magnetic specific heat contribution $\left(C_{m}\right)$ in $\mathrm{Ag}_{3} \mathrm{LiMn}_{2} \mathrm{O}_{6}$ was obtained by subtracting the lattice specific heat $C_{\text {lattice }}$. To estimate the $C_{\text {lattice }}$ of $\mathrm{Ag}_{3} \mathrm{LiMn}_{2} \mathrm{O}_{6}$, an isostructural material $\mathrm{Ag}_{3} \mathrm{LiTi}_{2} \mathrm{O}_{6}$ was prepared and its specific heat was measured in zero magnetic field, and prior to subtracting this from the $C_{p}(T)$ data of $\mathrm{Ag}_{3} \mathrm{LiMn}_{2} \mathrm{O}_{6}$ it was scaled with the $C_{p}(T)$ data of $\mathrm{Ag}_{3} \mathrm{LiMn}_{2} \mathrm{O}_{6}$. Initially, Bouvier scaling [27] was used to scale the lattice specific heat of $\mathrm{Ag}_{3} \mathrm{LiTi}_{2} \mathrm{O}_{6}$, which gives a correction factor $\left(\frac{\theta_{D}(\mathrm{Mn})}{\theta_{D}(\mathrm{Ti})}=0.988\right)$ to the temperature axis, where $\theta_{D}(\mathrm{Mn})$ and $\theta_{D}(\mathrm{Ti})$ are the Debye temperatures for the $\mathrm{Mn}$ and Ti compounds, respectively. However, this does not appear to reliably estimate the lattice specific heat of $\mathrm{Ag}_{3} \mathrm{LiMn}_{2} \mathrm{O}_{6}$. In fact, it was found to exceed the total specific heat of $\mathrm{Ag}_{3} \mathrm{LiMn}_{2} \mathrm{O}_{6}$. We then manually matched the specific heat of the nonmagnetic sample, in the high-temperature region, by rescaling its temperature axis by multiplying it by 1.085 . The magnetic specific heat $C_{m}$ is plotted in Fig. 5(b). The $C_{m}$ 


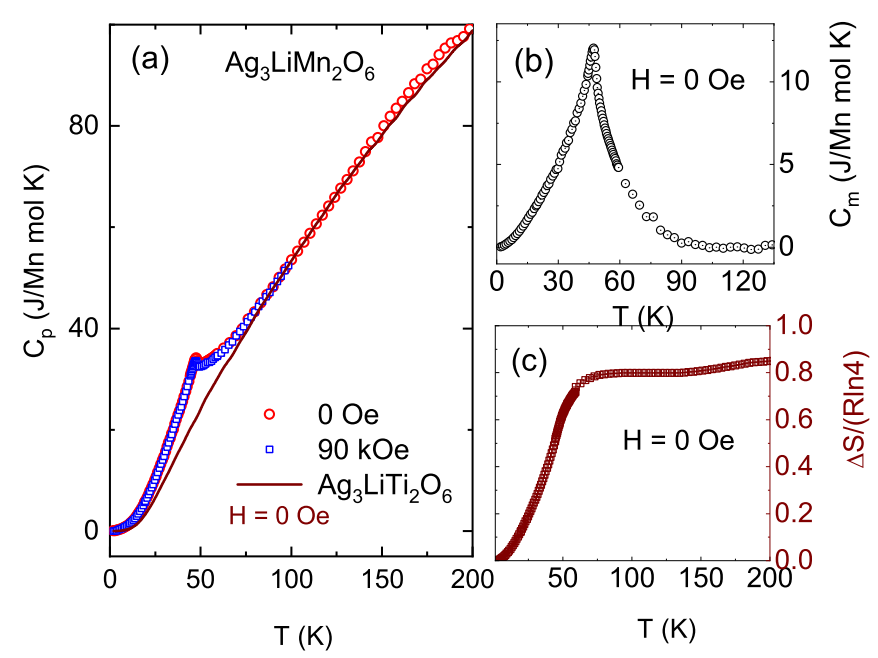

FIG. 5. (a) Specific heat of $\mathrm{Ag}_{3} \mathrm{LiMn}_{2} \mathrm{O}_{6}$ measured with $H=0$ Oe and $90 \mathrm{kOe}$ and nonmagnetic $\mathrm{Ag}_{3} \mathrm{LiTi}_{2} \mathrm{O}_{6}$ (solid brown line) at $H=0 \mathrm{Oe}$, used for the estimation of lattice contribution. (b) The magnetic specific heat, $C_{m}$, of $\mathrm{Ag}_{3} \mathrm{LiMn}_{2} \mathrm{O}_{6}$ at $H=0$ Oe. (c) Entropy change obtained at $H=0$ Oe normalized to $R \ln (4)$.

data clearly show a $\lambda$-like anomaly at $47 \mathrm{~K}$. The experimental magnetic entropy change $\Delta S$, estimated from the $C_{m}-T$ curve, is about $80 \% \pm 5 \%$ of the theoretical value of $11.52 \mathrm{~J} / \mathrm{Mn} \mathrm{mol}$ K for spin $S=3 / 2$, as can be seen in Fig. 5(c). The transition seen at $47 \mathrm{~K}$ approximately accounts for $55 \%$ entropy change while nearly the rest of the entropy is recovered above $70 \mathrm{~K}$. The recovery of a significant amount of entropy above the ordering temperature is probably suggestive of the presence of short-range magnetic correlations. One should recall that the intensity of the asymmetric peak seen in neutron diffraction measurements (see inset of Fig. 3) also does not immediately collapse to the peak recorded at $300 \mathrm{~K}$, indicating again that magnetic correlations survive at least up to $60 \mathrm{~K}$ and are in line with our specific heat analysis.

\section{ESR results}

To investigate the correlated magnetism originating as a result of interacting Mn moments, arranged in a honeycomb geometry, ESR measurements were performed as a function of temperature. In order to study the spin dynamics of $\mathrm{Ag}_{3} \mathrm{LiMn}_{2} \mathrm{O}_{6}$ and to get the evolution of the corresponding ESR parameters with temperature, the ESR line shape was analyzed. Typical ESR spectra of a powder sample of $\mathrm{Ag}_{3} \mathrm{LiMn}_{2} \mathrm{O}_{6}$ are shown in Fig. 6. All spectra consist of a single exchange-narrowed resonance line; i.e., any line splitting or inhomogeneous broadening is averaged out by the isotropic exchange interaction. The resonance line is well described by a Lorentz shape at resonance field $H_{\text {res }}$ with half-width at half maximum $\Delta H$ including the counter resonance at $-H_{\text {res }}$ and a small contribution of dispersion $(D)$ to the absorption (A) given by the $D / A$ ratio in the case of large linewidth as described in Ref. [28]. The results of ESR line shape fitting for some representative spectra are shown by solid lines in Fig. 6.

The ESR line is related to the manganese exchangecoupled system. The temperature dependencies of the res-
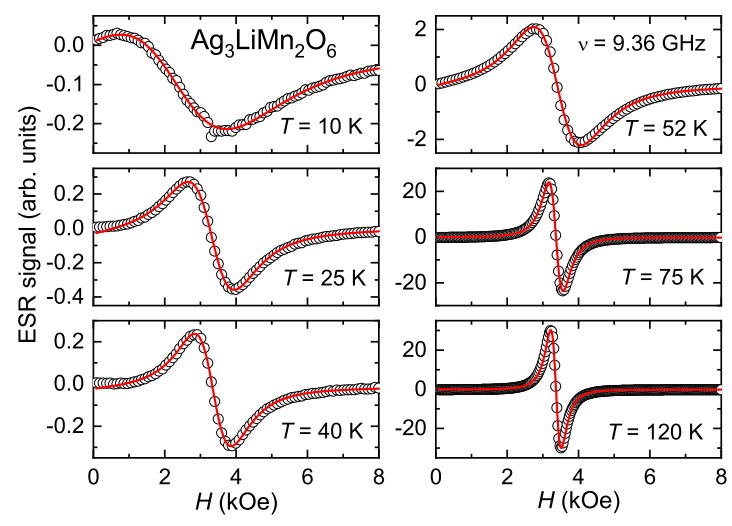

FIG. 6. ESR spectra of $\mathrm{Ag}_{3} \mathrm{LiMn}_{2} \mathrm{O}_{6}$ obtained at X-band frequency for different temperatures. Solid lines indicate Lorentz curves as described in the text.

onance field, the linewidth, the intensity, and the inverse intensity are summarized in Fig. 7. Starting with the resonance field [see Fig. 7 (a)] in the upper left panel, we obtain a $g$ value of $g=h v / \mu_{B} H_{\text {res }}=1.99$ at high temperature, which is typical for $\mathrm{Mn}^{4+}$ ions (spin $S=3 / 2$ ) in an octahedral ligand field with its half-filled $t_{2 g}$ triplet. Upon decreasing the temperature, the $g$ factor remains approximately constant down to a weak anomaly at $T_{\mathrm{N}}=48 \mathrm{~K}$ and then decreases upon further cooling followed by a divergence upon approaching zero temperature.

Focusing on the integrated intensity and its inverse representation shown in the panels on the right-hand side in Fig. 7, the data above $T_{\mathrm{N}}$ are perfectly described by a Curie-Weiss law $I=C_{1} /(T-\theta)$ with a Weiss temperature $\theta=-33 \mathrm{~K}$. Below $T_{\mathrm{N}}$ the temperature dependence of the intensity is well approximated by a pure Curie law $I=C_{2} / T$ (dashed line). As the integrated intensity corresponds to the spin susceptibility, it turns out from the comparison of the prefactors $C_{1}$ and $C_{2}$ that below $T_{\mathrm{N}}$ only $4 \%$ of the spins contribute to the ESR signal, i.e., weakly interacting spins, which are not involved in
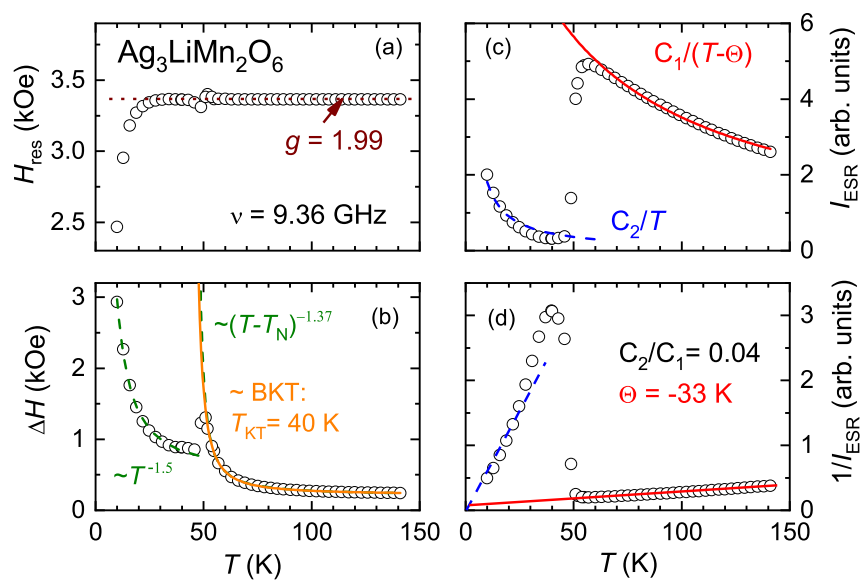

FIG. 7. The temperature dependence of the ESR parameters of $\mathrm{Ag}_{3} \mathrm{LiMn}_{2} \mathrm{O}_{6}$ : (a) resonance field (dashed line indicates $g=1.99$ ), (b) linewidth with critical behavior (dashed) and BKT scenario (solid), (c) ESR intensity, and (d) inverse intensity with Curie-Weiss law (solid) and low-temperature Curie law (dashed). 
the antiferromagnetic order, e.g., at the surface of the powder grains or at defect sites.

Turning finally to Fig. 7(b), the linewidth is found to depend strongly on temperature, indicating two different spindynamic regimes above and below $T_{\mathrm{N}}$. Starting from a value of about 200 Oe at high temperature, the linewidth is found to increase with decreasing temperature, diverging close to the antiferromagnetic phase transition with values larger than 1 kOe. Upon further cooling, the linewidth recovers to about 800 Oe but diverges again with values above 3 kOe below $4 \mathrm{~K}$.

The broadening of the ESR line upon approaching $T_{\mathrm{N}}$ may be treated in terms of critical behavior due to slowing down of spin fluctuations in the vicinity of an order-disorder transition. This causes the divergence of the spin correlation length, which in turn affects the spin-spin relaxation time of exchange-narrowed ESR lines resulting in the critical broadening, given by

$$
\Delta H(T)=\Delta H_{0}+A\left(\frac{T_{\mathrm{N}}}{T-T_{\mathrm{N}}}\right)^{\beta},
$$

where the first term $\Delta H_{0}$ describes the limiting-temperatureindependent linewidth for the exchange-narrowed regime, while the second term reflects the critical behavior with the critical exponent $\beta$. The dashed and the solid lines on the lower left panel in Fig. 7 represent a leastsquares fit of the linewidth data. The best fitting was obtained with the parameters $\Delta H_{0}=221(2) \mathrm{Oe}, A=13(2) \mathrm{kOe}, T_{N}=$ $45(1) \mathrm{K}$, and $\beta=1.37(5)$. The value of $T_{\mathrm{N}}$ obtained here is close to that from our heat capacity data. Below $T_{\mathrm{N}}$, the residual ESR signal diverges approximately with a power law $T^{-1.5}$.

Given the fact that the critical exponent of the divergence above $T_{\mathrm{N}}$ is significantly smaller than the expected value of 2.6 for the 2D Heisenberg antiferromagnetic model [29], the line broadening upon approaching $T_{\mathrm{N}}$ may be alternatively described in terms of a Berezinskii-Kosterlitz-Thouless (BKT) scenario like in the case of the honeycomb system $\mathrm{BaNi}_{2} \mathrm{~V}_{2} \mathrm{O}_{8}$ [30] with spin $S=1 \mathrm{Ni}^{2+}$. Indeed the temperature dependence of the linewidth is very well approximated by the expression

$$
\Delta H=A \exp \left[3 b /\left(T / T_{\mathrm{KT}}-1\right)^{0.5}\right]+\Delta H_{0}
$$

with the Kosterlitz-Thouless temperature $T_{\mathrm{KT}}=40(1) \mathrm{K}$, the parameter $b=0.83(6)$, the prefactor $A=11(2) \mathrm{Oe}$, and the residual linewidth $\Delta H_{0}=196(5) \mathrm{Oe}$, as shown in the lower left panel of Fig. 7. The BKT scenario indicates the spinspin relaxation via magnetic vortices, governed by the vortex correlation length, which diverges at $T_{\mathrm{KT}}$ due to vortexantivortex pairing. Originally this topological phase transition was derived for the XY model by Berezinskii [31] and by Kosterlitz and Thouless [32]. But later on it was shown [33] that already a weak anisotropy is enough to provide a BKT scenario. Concerning 2D spin $S=3 / 2$ antiferromagnets a BKT-type scenario was reported for the triangular lattice antiferromagnets $A \mathrm{CrO}_{2}$ with $A=\mathrm{H}, \mathrm{Li}, \mathrm{Na}, \mathrm{Cu}, \mathrm{Ag}, \mathrm{Pd}[34,35]$. In those Heisenberg antiferromagnets the frustration of the antiferromagnetic couplings gives rise to so-called $Z_{2}$ vortices [36]. Returning to the present Mn system, the obtained fit

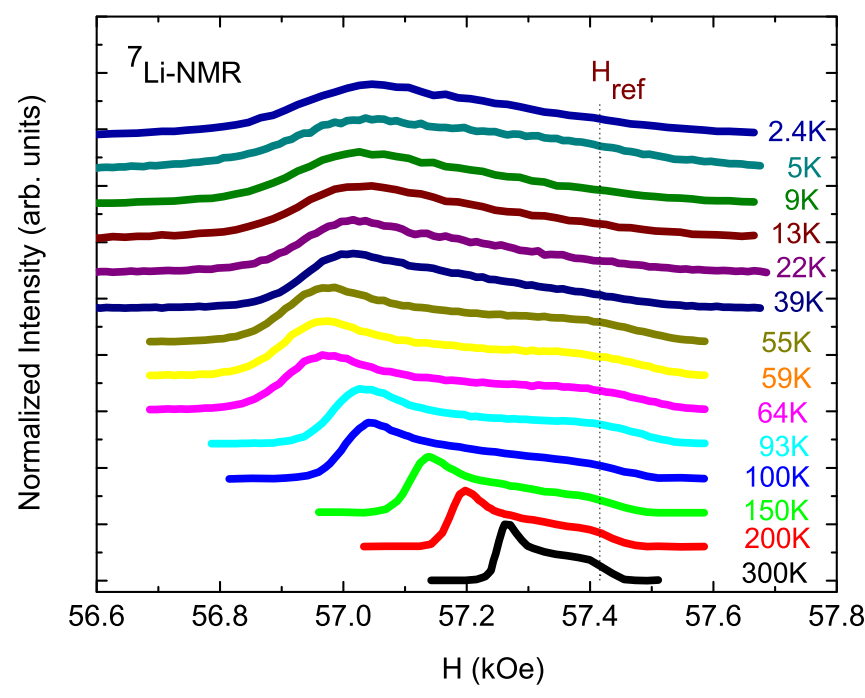

FIG. 8. ${ }^{7} \mathrm{Li}$ NMR spectra measured at different temperatures with fixed field $93.54 \mathrm{kOe}$ ( $T$ range $300-80 \mathrm{~K}$ ) and by sweeping the field at the transmitter frequency $95 \mathrm{MHz}$ ( $T$ range 2.4-93 K). The swept-field spectra were corrected for an average offset field of about 14 Oe because of a small offset in the field of the swept-field magnet compared to the fixed-field magnet. The fixed-field data were scaled to the swept-field data using an appropriate multiplier. The vertical dotted line indicates the ${ }^{7} \mathrm{Li}$ NMR reference field measured for an isostructural diamagnetic sample $\mathrm{Ag}_{3} \mathrm{LiTi}_{2} \mathrm{O}_{6}$.

parameters are comparable to those found in $\mathrm{BaNi}_{2} \mathrm{~V}_{2} \mathrm{O}_{8}$. The parameter $b<\pi / 2$ is in the range of theoretically sound values. The Kosterlitz-Thouless temperature of about $0.83 T_{\mathrm{N}}$ is typical for quasi-2D antiferromagnets, where the $3 \mathrm{D}$ antiferromagnetic order masks the Kosterlitz-Thouless transition. Using the relation [37]

$$
\frac{T_{\mathrm{N}}-T_{\mathrm{KT}}}{T_{\mathrm{KT}}}=\frac{4 b^{2}}{\left[\ln \left(J / J^{\prime}\right)\right]^{2}}
$$

derived for quasi-2D antiferromagnets (exchange constant $J$ ) with weak planar anisotropy and interplane coupling $J^{\prime}$ we obtain a ratio $J / J^{\prime} \approx 40$, if we use the experimental value of $b$ (or $\approx 800$, if we use $b=\pi / 2$ ). The appearance of longrange order (LRO) close to $\theta_{C W}$ in spite of the 2D nature might result from a renormalization of $T_{\mathrm{N}}$ due to a large in-plane antiferromagnetic (AFM) correlation length [38].

\section{E. NMR results}

In order to develop a microscopic understanding of the magnetic properties, ${ }^{7} \mathrm{Li}\left(I=3 / 2, \frac{\gamma}{2 \pi}=16.546 \mathrm{MHz} / \mathrm{T}\right) \mathrm{nu}-$ clear magnetic resonance (NMR) measurements were carried out on polycrystalline $\mathrm{Ag}_{3} \mathrm{LiMn}_{2} \mathrm{O}_{6}$.

The obtained ${ }^{7} \mathrm{Li}$ NMR spectra in the entire measured temperature range display a shoulder along with the main line; see Fig. 8. The main line is found to be broadened and shifted to the lower field side as a function of temperature with respect to the ${ }^{7} \mathrm{Li}$ NMR line measured for the isostructural diamagnetic sample $\mathrm{Ag}_{3} \mathrm{LiTi}_{2} \mathrm{O}_{6}$, while the shoulder remained almost unshifted upon lowering the temperature. The asymmetry (shoulder) in spectra could result from the anisotropy of hyperfine coupling. It must be noticed that this 


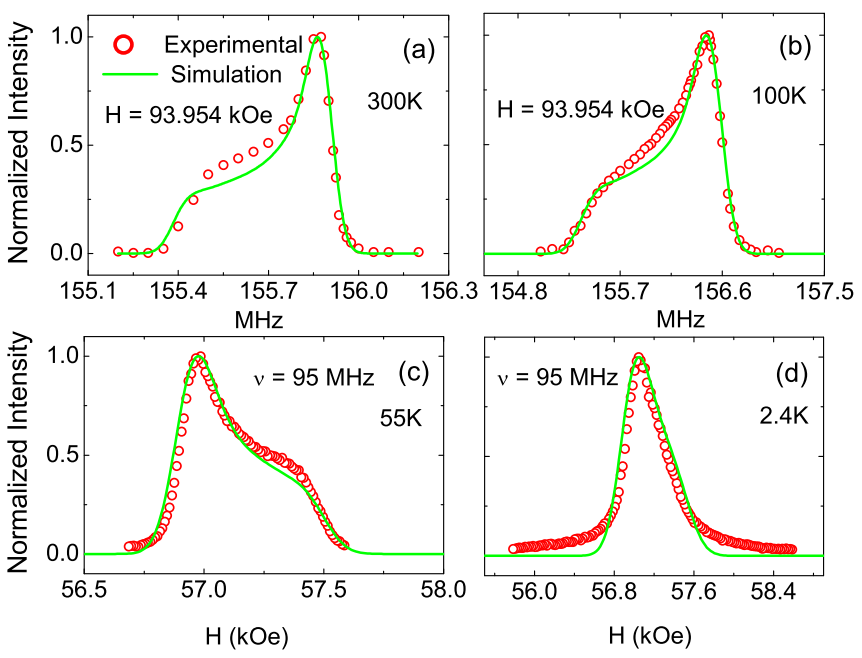

FIG. 9. Simulated ${ }^{7} \mathrm{Li}$ NMR spectra and experimentally collected ${ }^{7} \mathrm{Li}$ NMR spectra (for $\mathrm{Ag}_{3} \mathrm{LiMn}_{2} \mathrm{O}_{6}$ ) at different temperatures are shown by green solid lines and red open circles, respectively, for fixed-field data [(a) and (b)] and swept-field data [(c) and (d)].

asymmetry is most likely not from any chemical disorder between $\mathrm{Li} / \mathrm{Ru}$ as our $\mathrm{x}$-ray diffraction refinement results discard this possibility. Surprisingly, the asymmetry in spectra was also seen in the isostructural material $\mathrm{Ag}_{3} \mathrm{LiRu}_{2} \mathrm{O}_{6}$ [9]. Interestingly, the hydrogenated analog of $\mathrm{Ag}_{3} \mathrm{LiIr}_{2} \mathrm{O}_{6}$, i.e., $\mathrm{H}_{3} \mathrm{LiIr}_{2} \mathrm{O}_{6}$, does not show significant asymmetry in ${ }^{1} \mathrm{H}$ NMR line shape [39].

We analyzed the powder-averaged ${ }^{7} \mathrm{Li}$ NMR spectra of $\mathrm{Ag}_{3} \mathrm{LiMn}_{2} \mathrm{O}_{6}$, similar to the previously studied material $\mathrm{Ag}_{3} \mathrm{LiRu}_{2} \mathrm{O}_{6}$, by fitting the spectra to a combination of the anisotropic shift parameters $K_{\text {iso }}$ and $K_{\text {aniso. }}$. A few representative simulated patterns are shown in Fig. 9. The extracted $K_{\text {iso }}$ from ${ }^{7} \mathrm{Li}$ NMR spectra in the temperature range $300-3 \mathrm{~K}$ as a function of temperature is shown in Fig. 10. The $K_{\text {iso }}$ data follow the bulk susceptibility data down to about $50 \mathrm{~K}$ and

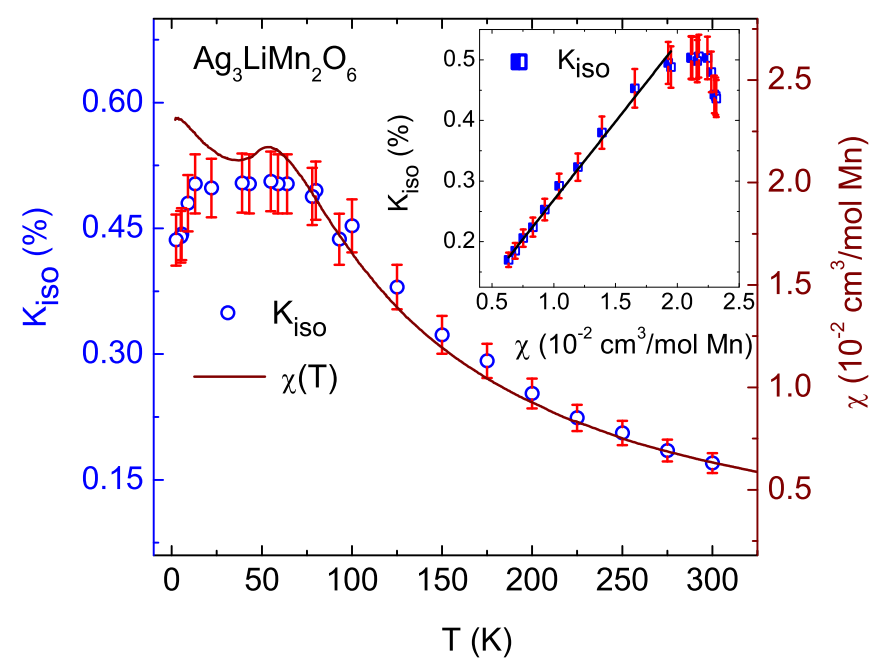

FIG. 10. The left $y$ axis shows the $K_{\text {iso }}$ (blue open circles) as a function of temperature and the right $y$ axis depicts the bulk susceptibility (solid line) data for $\mathrm{Ag}_{3} \mathrm{LiMn}_{2} \mathrm{O}_{6}$. The inset shows a plot of $K_{\text {iso }}$ versus $\chi$ with temperature as an implicit parameter.

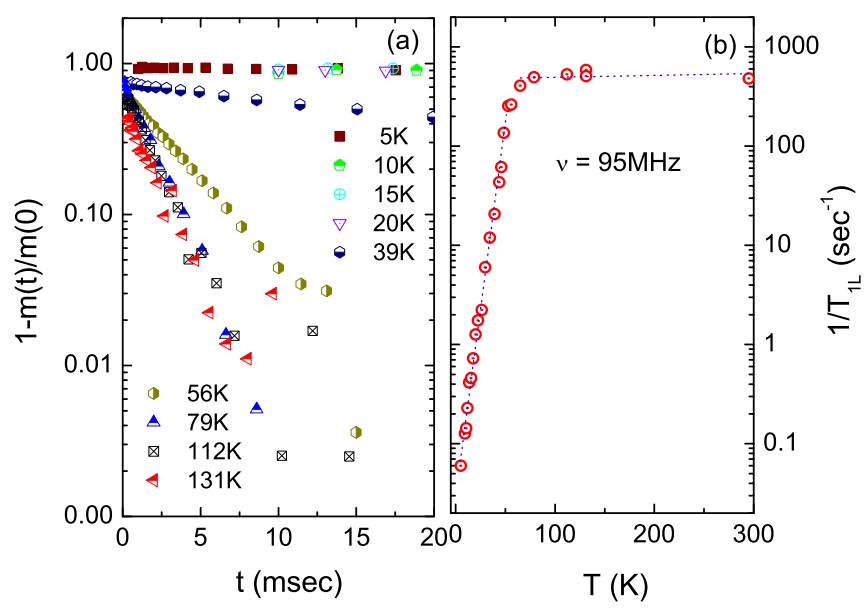

FIG. 11. (a) Recovery of the ${ }^{7} \mathrm{Li}$ longitudinal nuclear magnetization as a function of delay time at a few representative temperatures. (b) Spin-lattice relaxation rate as a function of temperature for the long component $T_{1 L}$ (smaller rate).

show an anomaly around the ordering temperature. The low- $T$ deviation of the bulk susceptibility from the NMR shift could be the result of some extrinsic impurity contribution or intrinsic defects. The hyperfine coupling constant $\left(A_{h f}\right)$ and the chemical shift obtained from the $K-\chi$ plot (inset of Fig. 10) yield $1.45 \pm 0.04 \mathrm{kOe} / \mu_{B}$ and $0.009(6) \%$, respectively.

The ${ }^{7} \mathrm{Li}$ NMR spin-lattice relaxation rate $\left(1 / T_{1}\right)$ measurements were performed with the intention to study the lowenergy spin dynamics or to probe the $q$-averaged dynamical susceptibility of $\mathrm{Ag}_{3} \mathrm{LiMn}_{2} \mathrm{O}_{6}$ in the temperature range 2 $300 \mathrm{~K}$ at the transmitter frequency $95 \mathrm{MHz}$. The saturation recovery method was employed to measure the $1 / T_{1}$ data. In order to deduce the spin-lattice relaxation time from the measured data, the data were fitted to a combination of two exponential decays given by the equation:

$$
1-m(t) / m(0)=A \exp \left(-x / T_{1 L}\right)+B \exp \left(-x / T_{1 S}\right),
$$

where $T_{1 L}$ and $T_{1 S}$ are the long and short components of spinlattice relaxation time with $A$ and $B$ being constants.

Figure 11(a) depicts a plot for ${ }^{7} \mathrm{Li}$ nuclear magnetization saturation recoveries at selected temperatures for $\mathrm{Ag}_{3} \mathrm{LiMn}_{2} \mathrm{O}_{6}$. A two-component spin-lattice relaxation was also observed in $\mathrm{Ag}_{3} \mathrm{LiRu}_{2} \mathrm{O}_{6}$. This could result from an incomplete saturation of the NMR line leading to spectral diffusion and an initial fast recovery. The long component of the spin-lattice relaxation rate $\left(1 / T_{1 L}\right)$ for $\mathrm{Ag}_{3} \mathrm{LiMn}_{2} \mathrm{O}_{6}$ is illustrated in Fig. 11(b). The $1 / T_{1 L}$ data in the temperature range $300-60 \mathrm{~K}$ do not show any variation as a function of temperature; however, below about $60 \mathrm{~K}, 1 / T_{1 L}$ starts to deviate from this behavior. At the antiferromagnetic ordering temperature, the $1 / T_{1 L}$ data should have also exhibited a distinct anomaly, but no peak was seen and a smooth decrease was noticed in $1 / T_{1 L}$ data in the temperature range $2-50 \mathrm{~K}$. The absence of the signature of any AFM order in the $1 / T_{1 L}$ data most likely results from a cancellation of the antiferromagnetic fluctuations at the Li position because of its symmetric position in a honeycomb network of $\mathrm{Mn}$ atoms. So, because of the 2D symmetric arrangement of $\mathrm{Mn}$ atoms around the $\mathrm{Li}$ atom, which sits in the middle of the 
honeycomb lattice, the $1 / T_{1 L}$ data measured for ${ }^{7} \mathrm{Li}$ nuclei will not sense fluctuations in the hyperfine field perpendicular to the applied field. Thus one, in principle, does not expect to see any sharp kink or any critical divergence in the $1 / T_{1 L}$ data. This finding further strengthens the idea of a symmetric crystallographic position of $\mathrm{Li}$ atom, $2 \mathrm{a}(0,0,0)$, with respect to its surrounding $\mathrm{Mn}$ atoms in a unit cell and also an absence of $\mathrm{Li} / \mathrm{Mn}$ site disorder. The $1 / T_{1 L}$ becomes very long with decrease in temperature as static order develops and there is absence of any fluctuations.

\section{F. Electronic structure calculation}

In order to identify the dominant exchange paths and the relevant spin Hamiltonian of the system we have performed first-principles electronic structure calculation using VASP. The $\mathrm{MnO}_{6}$ octahedral units that host magnetism form an edge-shared honeycomb geometry in the $a b$ plane with Li ions at the center of the honeycomb. Upon relaxation, the distances of three nearest-neighbor (NN) Mn atoms become $2.926 \AA$ and $2.932 \AA$ with coordination two and one, respectively. The $\mathrm{Mn}-\mathrm{O}-\mathrm{Mn}$ angles in the respective $\mathrm{NN}$ paths are $102.33^{\circ}$ and $102.61^{\circ}$. The $\mathrm{MnO}_{6}$ octahedra have a monoclinic distortion and hence the $\mathrm{Mn}-\mathrm{O}$ bond lengths are unequal $(1.876 \AA$ to $1.878 \AA$ ) as also the $\mathrm{Mn}-\mathrm{O}-\mathrm{Mn}$ bond angles $\left(77.68^{\circ}\right.$ to $\left.95.05^{\circ}\right)$.

In order to understand the basic electronic structure, we have first carried out non-spin-polarized calculations enforcing the spin degeneracy. The octahedral crystal field breaks the degeneracy of $5 d$ states of $\mathrm{Mn}$ atoms into triply degenerate $t_{2 g}$ and doubly degenerate $e_{g}$ states that get further split due to monoclinic distortion maintaining crystal field splitting between $t_{2 g}$ and $e_{g}$ states to be $2.5 \mathrm{eV}$. The calculations reveal that the Mn $t_{2 g}$ states are half filled consistent with the $\mathrm{Mn}^{4+}\left(d^{3}\right)$ configuration resulting in a metallic solution.

Next we have performed spin-polarized calculations with FM arrangement of Mn spins within the GGA approximation. A plot of the spin-polarized density of states (DOS) for the FM configuration shown in Fig. 12(a) reveals that in the majorityspin channel the Mn- $d t_{2 g}$ states are completely filled and the minority $t_{2 g}$ states are complete empty with an exchange splitting of about $0.62 \mathrm{eV}$. The $e_{g}$ states in both the spin channels are completely empty. In the FM calculation, the total moment per formula unit, containing $2 \mathrm{Mn}$ atoms, is calculated to be $6.0 \mu_{B}$, which further supports the $4+$ charge state of $\mathrm{Mn}$ and is also consistent with the experimentally calculated value of $\mu_{\text {eff }}\left(4.46 \pm 0.33 \mu_{B}\right)$. FM calculation gives the magnetic moment per Mn site to be $2.68 \mu_{B}$, as the rest of the moment lies in the ligand sites $\left(0.04 \mu_{B} / \mathrm{O}\right)$ due

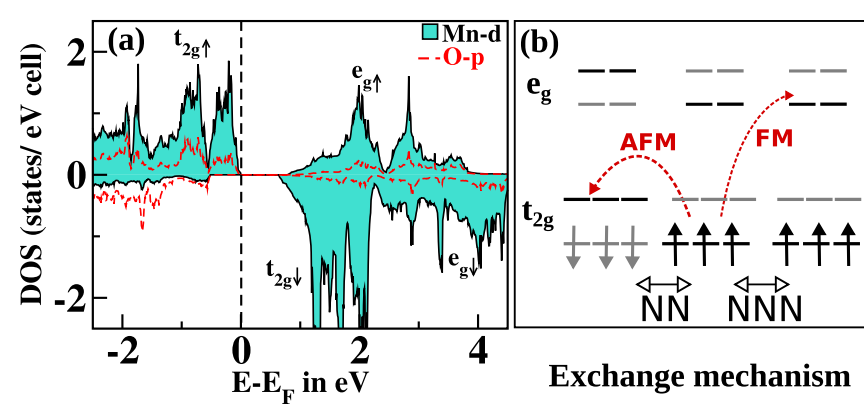

FIG. 12. (a) The orbital-decomposed spin-polarized DOS. The cyan shaded region and the red dotted lines, respectively, show the contribution of Mn- $d$ and O-p states per atom. (b) The schematic diagram for the NN AFM and NNN FM exchange interaction mechanism.

to substantial hybridization. Inclusion of Coulomb correlation $(U)$ further increases the exchange splitting $(0.99 \mathrm{eV})$ and localizes the $d$ orbitals, which essentially increases the moment of $\operatorname{Mn}\left(2.95 \mu_{B} / \mathrm{Mn}\right)$.

In order to identify the nature of the ground state we have calculated the energies of different possible collinear magnetic configurations, namely FM, AFM-1, AFM-2, and AFM-3. The results are shown in Table II. In the AFM-1 configuration, all nearest-neighbor interactions $\left(J_{1}\right.$ and $\left.J_{2}\right)$ are antiferromagnetic, similarly to the ground state AFM solution of a bipartite lattice. In the AFM- 2 configuration, among the two types of NN interactions, $J_{1}$ is AFM while $J_{2}$ is ferromagnetic. On the contrary the AFM-3 configuration has FM $J_{1}$ and AFM $J_{2}$ interactions. From Table II it is evident that AFM-1 is the lowest energy configuration with $\mathrm{NN}$ interactions antiferromagnetic.

Now for a quantitative estimate of Mn intersite exchange strengths, we have calculated symmetric exchange interactions with the "four state" method based on the total energy of the system with few collinear spin alignments. If the magnetism in the system is fully described by the Heisenberg Hamiltonian, the energy for such a spin pair can be written as follows [40]:

$$
E=-J_{12} \boldsymbol{S}_{1} \cdot \boldsymbol{S}_{2}+\boldsymbol{S}_{1} \cdot \boldsymbol{h}_{1}+\boldsymbol{S}_{2} \cdot \boldsymbol{h}_{2}+E_{\text {all }}+E_{0},
$$

where $J_{i j}$ is the symmetric exchange coupling along the bond that connects spin pair $i$ and $j . h_{1}=-\sum_{i \neq 1,2} J_{1 i} S_{i}$, $\boldsymbol{h}_{2}=-\sum_{i \neq 1,2} J_{2 i} \boldsymbol{S}_{i}, E_{\text {all }}=-\sum_{i, j \neq 1,2} J_{i j} \boldsymbol{S}_{i} \cdot \boldsymbol{S}_{j}$, and $E_{0}$ contains all other nonmagnetic energy contributions. The second (third) term in Eq. (4) corresponds to the coupling of the spin 1 (2) with all other spins in the unit cell except spin $2(1) ; E_{\text {all }}$

TABLE II. Variation of the magnetic moments (in the GGA $+U$ calculation) on the various ions for different ordering arrangements and their corresponding energies are tabulated here.

\begin{tabular}{lcccc}
\hline \hline $\begin{array}{l}\text { Magnetic } \\
\text { Configuration }\end{array}$ & $\begin{array}{c}\text { (Total Moment)/f.u. } \\
\left(\mu_{B}\right)\end{array}$ & $\begin{array}{c}\text { Moment/Mn } \\
\left(\mu_{B}\right)\end{array}$ & $\begin{array}{c}\text { Gap } \\
(\mathrm{eV})\end{array}$ & $\begin{array}{c}\Delta E / \text { f.u. } \\
(\mathrm{meV})\end{array}$ \\
\hline AFM-1 & 0.0 & 2.88 & 1.54 & 0.0 \\
AFM-2 & 0.0 & 2.91 & 1.33 & 36.6 \\
AFM-3 & 0.0 & 2.89 & 1.47 & 24.6 \\
FM & 6.0 & 2.95 & 0.99 & 35.5 \\
\hline \hline
\end{tabular}




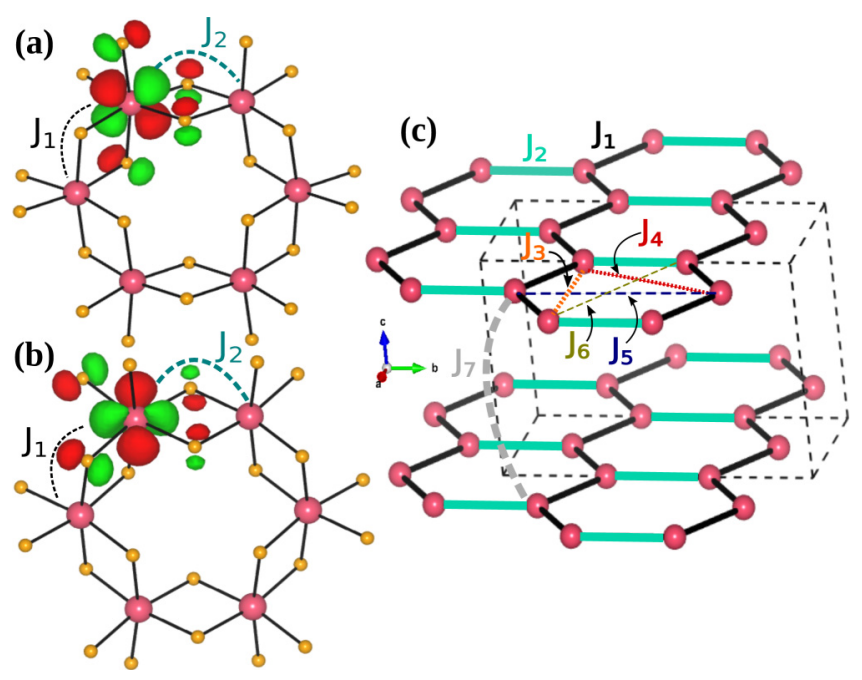

FIG. 13. (a) and (b) are two different Wannier orbitals connecting NN Mn atoms. (c) Different symmetric exchange interactions corresponding to neighbor distances. $\mathrm{NN} J_{1}$ and $J_{2}$ interactions are marked with black and cyan solid lines. NNN interactions $J_{3}$ and $J_{4}$, 3rd neighbors $J_{5}$ and $J_{6}$, and interplane interaction are denoted with orange, red, brown, blue, and gray dotted lines, respectively.

characterizes the exchange couplings between all spins in the unit cell apart from spins 1 and 2. The exchange interaction strength between sites 1 and 2 obtained with total energy of four collinear spin alignments [such as $\left(1_{\uparrow} 2_{\uparrow}\right),\left(1_{\uparrow} 2_{\downarrow}\right)$, $\left.\left(1_{\downarrow} 2_{\uparrow}\right),\left(1_{\downarrow} 2_{\downarrow}\right)\right]$ has the expression [40]

$$
J_{12}=-\frac{E_{\uparrow \uparrow}+E_{\downarrow \downarrow}-E_{\uparrow \downarrow}-E_{\downarrow \uparrow}}{4 S^{2}} .
$$

The first (second) suffix of energy $(E)$ tells the spin state of site 1 (2). The obtained symmetric exchange interactions are $J_{1}=-2.59 \mathrm{meV}(\mathrm{AFM}), J_{2}=-2.28 \mathrm{meV}$ (AFM), $J_{3}=$ $0.54 \mathrm{meV} \quad(\mathrm{FM}), J_{4}=0.69 \mathrm{meV} \quad(\mathrm{FM}), J_{5}=-0.16 \mathrm{meV}$ (AFM), and $J_{6}=-0.15 \mathrm{meV}$ (AFM), and the respective exchange paths are shown in Fig. 13(c). The possible mechanism of spin-conserved exchange couplings for the NN AFM exchange interactions $\left(J_{1}\right.$ and $\left.J_{2}\right)$ and FM second neighbors $\left(J_{3}\right.$ and $\left.J_{4}\right)$ are shown in Fig. 12(b). For NN AFM alignments, exchange splitting and intersite hoppings are the key parameters, while for the FM arrangement, spins have to overcome the crystal field splitting with nearest-neighbor superexchange hopping to obey the Hund's coupling. The strongest NN interactions along with the finite (though small) farther-neighbor interactions results in the long-range AFM ordering in the system. The Wannier function plots in Figs. 13(a) and 13(b) clearly show that the NN interactions via the $\mathrm{Mn}-\mathrm{O}-\mathrm{Mn}$ superexchange path are dominant. The substantial deviation of Mn-O-Mn angle(s) $\left(\sim 102^{\circ}\right)$ from $\frac{\pi}{2}$ along paths $J_{1(2)}$ favors an antiferromagnetic alignment in accordance with Goodenough-Kanamori-Anderson rules [41-44], which is consistent with our results. To check the strength of interlayer coupling along the $c$ direction we have fixed the static intraplane magnetic arrangement to AFM-1 and applied the above mentioned "four state" method to calculate the interlayer exchange $J_{7}$. The interplanar coupling strength is estimated to be FM in nature with magnitude $0.14 \mathrm{meV}$. The theoretically calculated $\theta_{C W}$ from these exchange strengths turns out to be $-54.8 \mathrm{~K}$, which is very close to the experimentally obtained value, $-51 \mathrm{~K}$. The ratio of interplanar and NN intraplanar interaction $\left(\frac{J_{N N}}{J_{\text {intẹr }}}\right)$ is nearly 19.0, which suggests that the magnetic network is primarily $2 \mathrm{D}$ in nature.

\section{CONCLUSIONS}

In summary, the new honeycomb material $\mathrm{Ag}_{3} \mathrm{LiMn}_{2} \mathrm{O}_{6}$ has been studied using $\mathrm{x}$-ray diffraction, neutron diffraction, magnetization, specific heat, ESR, and NMR measurements and first-principles calculations. An asymmetric peak, a signature of superstructure, is seen in both the x-ray and neutron diffraction measurements, and it is found to grow in intensity below about $50 \mathrm{~K}$ before saturating at lower temperatures. This suggests magnetic ordering of the honeycomb lattice. The susceptibility measurements carried out for $\mathrm{Ag}_{3} \mathrm{LiMn}_{2} \mathrm{O}_{6}$ show an anomaly around $50 \mathrm{~K}$ and the presence of antiferromagnetic interactions $\left(\theta_{C W} \sim-51 \mathrm{~K}\right)$ among Mn moments. The neutron diffraction data measured down to $2 \mathrm{~K}$ clearly show the onset of magnetic ordering below $50 \mathrm{~K}$, in agreement with the anomaly observed in the susceptibility data of $\mathrm{Ag}_{3} \mathrm{LiMn}_{2} \mathrm{O}_{6}$. The heat capacity measurements further support long-range magnetic order in $\mathrm{Ag}_{3} \mathrm{LiMn}_{2} \mathrm{O}_{6}$ by exhibiting a sharp peak in the measured specific heat around $47 \mathrm{~K}$. The entropy change inferred from the heat capacity data suggests that the system needs to be heated to nearly 1.6 times the ordering temperature to recover the full entropy. This suggests that 2D magnetic correlations start building up at high temperature; upon cooling, a fraction of the entropy is already lost when the sample locks into LRO around $50 \mathrm{~K}$. Our local probe ${ }^{7} \mathrm{Li}$ NMR spectra measurements done on the powder samples, which basically measure the static susceptibility, also support thermodynamic measurements by exhibiting a clear anomaly in the measured ${ }^{7} \mathrm{Li}$ NMR shift. The ${ }^{7} \mathrm{Li}$ spin-lattice relaxation rate, which is a measure of the $q$-averaged dynamical susceptibility, does not show a peak as observed in other measurements, presumably because of the cancellation of antiferromagnetic fluctuations at the center $(\mathrm{Li}$ site) of the hexagon, also implying that the structure remains that of a regular honeycomb with $\mathrm{Li}$ sitting at the centers of the honeycomb network. However, ${ }^{7} \mathrm{Li} 1 / T_{1}$ too shows a sharp decrease of nearly four orders of magnitude below about $50 \mathrm{~K}$, indicating the quenching of magnetic fluctuations due to the onset of magnetic order. Taken together, our thermodynamic and ${ }^{7} \mathrm{Li} \mathrm{NMR}$ shift measurements evidence the emergence of an ordered ground in the $3 d$ honeycomb material $\mathrm{Ag}_{3} \mathrm{LiMn}_{2} \mathrm{O}_{6}$. Our experimental results are corroborated by first-principles electronic structure calculations. Our $a b$ initio calculations find that the $\mathrm{NN}$ interactions $J_{1(2)}$ are antiferromagnetic. The farther-neighbor interactions also do not give rise to any frustration. Thus, in the case of a dominant Heisenberg term (as might be expected in this case), and even a small interplanar coupling (as per our calculations), the honeycomb system displays long-range order as seen here. The manifestation of $2 \mathrm{D}$ effects in $\mathrm{Ag}_{3} \mathrm{LiMn}_{2} \mathrm{O}_{6}$ is seen from the analysis of the $T$ dependence of the ESR linewidth above the transition temperature. We obtained a Kosterlitz-Thouless temperature of about $0.83 T_{\mathrm{N}}$, which is typical for quasi-2D antiferromagnets. The weak interplanar coupling is sufficient 
to lock the system into $3 \mathrm{D}$ order, which then masks the Kosterlitz-Thouless transition.

\section{ACKNOWLEDGMENTS}

This work is partially based on experiments performed at the Swiss spallation neutron source SINQ, Paul Scherrer Institute, Villigen, Switzerland. We thank the Department of Science and Technology (DST), Government of India, for financial support through the BRICS project Helimagnets. I.D. thanks DST, Government of India, and TRC for financial support. R.K. acknowledges CSIR, India, and IRCC, IIT
Bombay, for awarding him fellowships for the completion of this work. P.M.E. acknowledges CSIR, India, for providing financial support under the CSIR-SRF Fellowship [Grant No. 31/52(14)2k17]. A.V.M. would like to thank the Alexander von Humboldt Foundation for financial support during the stay at the University of Augsburg. The work of R.E. was done within the framework of fundamental research AAAA-A18118030690040-8 of FRC Kazan Scientific Center of RAS. A.A.G. acknowledges the financial support from the RFBR Grant No. 17-52-80036. Additionally, we kindly acknowledge support from the German Research Society (DFG) via TRR80 (Augsburg and Munich).
[1] R. D. Shannon, D. B. Rogers, and C. T. Prewitt, Inorg. Chem. 10, 713 (1971).

[2] H. Kawazoe, M. Yasukawa, H. Hyodo, M. Kurita, H. Yanagi, and H. Hosono, Nature (London, UK) 389, 939 (1997).

[3] E. Wawrzyńska, R. Coldea, E. M. Wheeler, T. Sörgel, M. Jansen, R. M. Ibberson, P. G. Radaelli, and M. M. Koza, Phys. Rev. B 77, 094439 (2008).

[4] M. Poienar, F. Damay, C. Martin, V. Hardy, A. Maignan, and G. André, Phys. Rev. B 79, 014412 (2009).

[5] A. Maignan, C. Martin, R. Frésard, V. Eyert, E. Guilmeau, S. Hébert, M. Poienar, and D. Pelloquin, Solid State Commun. 149, 962 (2009).

[6] M. A. Marquardt, N. A. Ashmore, and D. P. Cann, Thin Solid Films 496, 146 (2006).

[7] S. A. J. Kimber, C. D. Ling, D. Jonathan, P. Morris, A. Chemseddine, P. F. Henry, and D. N. Argyriou, J. Mater. Chem. 20, 8021 (2010).

[8] K. Ramesha, A. S. Prakash, M. Sathiya, G. Madras, and A. K. Shukla, Mater. Sci. Eng. B 176, 141 (2011).

[9] R. Kumar, T. Dey, P. M. Ette, K. Ramesha, A. Chakraborty, I. Dasgupta, J. C. Orain, C. Baines, S. Tóth, A. Shahee, S. Kundu, M. Prinz-Zwick, A. A. Gippius, N. Büttgen, P. Gegenwart, and A. V. Mahajan, Phys. Rev. B 99, 054417 (2019).

[10] V. Todorova, A. Leineweber, L. Kienle, V. Duppel, and M. Jansen, J. Solid State Chem. 184, 1112 (2011).

[11] G. Khaliullin, Phys. Rev. Lett. 111, 197201 (2013).

[12] O. N. Meetei, W. S. Cole, M. Randeria, and N. Trivedi, Phys. Rev. B 91, 054412 (2015).

[13] C. Svoboda, M. Randeria, and N. Trivedi, Phys. Rev. B 95, 014409 (2017).

[14] S. Trebst, arXiv:1701.07056.

[15] T.-C. Wei, I. Affleck, and R. Raussendorf, Phys. Rev. Lett. 106, 070501 (2011).

[16] M. Koch-Janusz, D. I. Khomskii, and E. Sela, Phys. Rev. Lett. 114, 247204 (2015).

[17] J. P. Perdew, K. Burke, and M. Ernzerhof, Phys. Rev. Lett. 77, 3865 (1996).

[18] G. Kresse and J. Hafner, Phys. Rev. B 47, 558 (1993).

[19] G. Kresse and J. Furthmüller, Phys. Rev. B 54, 11169 (1996).

[20] P. E. Blöchl, Phys. Rev. B 50, 17953 (1994).

[21] G. Kresse and D. Joubert, Phys. Rev. B 59, 1758 (1999).
[22] S. L. Dudarev, G. A. Botton, S. Y. Savrasov, C. J. Humphreys, and A. P. Sutton, Phys. Rev. B 57, 1505 (1998).

[23] A. A. Mostofi, J. R. Yates, G. Pizzi, Y.-S. Lee, I. Souza, D. Vanderbilt, and N. Marzari, Comput. Phys. Commun. 185, 2309 (2014).

[24] B. E. Warren, Phys. Rev. 59, 693 (1941).

[25] J. R. Carvajal, Abstracts of the Satellite Meeting on Powder Diffraction of the XV Congress of the IUCr, 1990.

[26] Z. Zeng, M. Greenblatt, and M. Croft, Phys. Rev. B 59, 8784 (1999).

[27] M. Bouvier, P. Lethuillier, and D. Schmitt, Phys. Rev. B 43, 13137 (1991).

[28] J. P. Joshi and S. Bhat, J. Magn. Reson. 168, 284 (2004).

[29] H. Benner and J. P. Boucher, Magnetic Properties of Layered Transition Metal Compounds, edited by L. J. de Jongh (Kluwer Academic Publishers, Dordrecht, 1990), pp. 323-378 .

[30] M. Heinrich, H.-A. Krug von Nidda, A. Loidl, N. Rogado, and R. J. Cava, Phys. Rev. Lett. 91, 137601 (2003).

[31] V. L. Berezinskii, Zh. Eksp. Teor. Fiz. 59, 907 (1971) [JETP 32, 493 (1971)].

[32] J. M. Kosterlitz and D. J. Thouless, J. Phys. C: Solid State Phys. 6, 1181 (1973).

[33] A. Cuccoli, T. Roscilde, R. Vaia, and P. Verrucchi, Phys. Rev. Lett. 90, 167205 (2003).

[34] M. Hemmida, H.-A. Krug von Nidda, and A. Loidl, J. Phys. Soc. Jpn. 80, 053707 (2011).

[35] L. K. Alexander, N. Büttgen, R. Nath, A. V. Mahajan, and A. Loidl, Phys. Rev. B 76, 064429 (2007).

[36] H. Kawamura and S. J. Miyashita, J. Phys. Soc. Jpn. 53, 4138 (1984).

[37] S. T. Bramwell and P. C. W. Holdsworth, J. Phys.: Condens. Matter 5, L53 (1993).

[38] S. Chakravarty, B. I. Halperin, and D. R. Nelson, Phys. Rev. Lett. 60, 1057 (1988).

[39] K. Kitagawa, T. Takayama, Y. Matsumoto, A. Kato, R. Takano, Y. Kishimoto, S. Bette, R. Dinnebier, G. Jackeli, and H. Takagi, Nature (London, UK) 554, 341 (2018).

[40] H. J. Xiang, E. J. Kan, S.-H. Wei, M.-H. Whangbo, and X. G. Gong, Phys. Rev. B 84, 224429 (2011).

[41] P. W. Anderson, Phys. Rev. 79, 350 (1950).

[42] J. B. Goodenough, Phys. Rev. 100, 564 (1955).

[43] J. B. Goodenough, J. Phys. Chem. Solids 6, 287 (1958).

[44] J. Kanamori, J. Phys. Chem. Solids 10, 87 (1959). 